\title{
DISTRIBUTION OF ZEROS AND LIMIT BEHAVIOR OF SOLUTIONS OF DIFFERENTIAL EQUATIONS ${ }^{1}$
}

\author{
H. GUGGENHEIMER
}

\begin{abstract}
Elementary arguments of projective geometry are used to obtain information about the limit behavior of solutions of ordinary linear differential equations if certain distributions of zeros do not occur.
\end{abstract}

The connection between the projective differential geometry of curves and ordinary differential equations was first explored by G. D. Birkhoff [2] and used recently by the author [3], [4] and F. Neuman [6]. In the present note, the same method is applied to study the behavior in the large of solutions of differential equations for which certain distributions of zeros do not occur. The definitions and notations of the survey of $J$. H. Barrett [1] are used without further notice.

A vector $\left(y_{1}(t), \ldots, y_{n}(t)\right)$ of linearly independent solutions of an $n$th order equation $y^{(n)}+\sum p_{i}(t) y^{(n-i)}=0$ represents a curve $\mathbf{y}(t)=$ $\left(y_{1}(t): y_{2}(t): \cdots: y_{n}(t)\right)$ in real projective $(n-1)$-space. A change of basis induces a linear transformation of the vector and therefore a projective transformation of $\mathbf{y}(t)$. Since the Wronskian is different from zero at all points, the curve $y(t)$ is regular, without inflection points and locally on the boundary of its convex hull: in each affine subspace of projective space the curve is locally convex in the sense of B. Segre [8], locally convex in the usual sense if $n=3$. A projective transformation can also be viewed as a change of the fundamental simplex. A solution $y_{i}(t)$ with a given distribution of zeros is induced by $\mathbf{y}(t)$ in a coordinate system for which the curve intersects a fixed hyperplane $y_{i}=0$ a given number of times with given orders of contact. All the results of the present note follow from this remark. All limits taken are in $\mathbf{R} \cup\{\infty\} \cup\{-\infty\}$. All equations are defined on $\left(t_{0}, \infty\right)$ with unique solutions of the initial value problem.

1. If $r_{21}(u)=\infty$ for a third order equation, i.e., no solution with a double zero at $t_{0} \geqslant u$ has a zero for $t>t_{0}$, then the tangent to $\mathbf{y}(t)$ at any $t_{0} \geqslant u$ has no intersection with the curve for $t>t_{0}$. If we take the tangent at $\mathbf{y}(u)$ as line at infinity, $\mathbf{y}(t)$ for $t>t_{0}$ is a locally convex Jordan arc in the corresponding affine plane and hence either tends to a limit in the projective plane or to a closed convex limit cycle in the affine plane.

Definition. A curve in the projective plane is projectively convex if it is the

Received by the editors February 27, 1976.

AMS (MOS) subject classifications (1970). Primary 34C10.

${ }^{1}$ Research partially supported by NSF Grant GP-27960. 
image of a convex curve in an affine plane.

With this definition, the result of our argument is

PROPOSITION 1. If $r_{21}(u)=\infty$ for a third order linear differential equation then either $\lim _{t \rightarrow \infty} y_{i}(t) / y_{j}(t)$ exists for any two solutions of the equation or $\mathbf{y}(t)=\left(y_{1}(t): y_{2}(t): y_{3}(t)\right)$ is asymptotic to a closed, projectively convex curve.

A number of special results can be obtained by prescribing the possible behavior according to Proposition 1. A sample is given by the next result.

Proposition 2. If $r_{12}=\infty$ and the solution specified up to linear dependence by $y(u)=y^{\prime}(u)=0$ does not tend to a limit 0 for $t \rightarrow \infty$ then for $t^{*}>u$ there exists $a$ basis of the solutions of the equations for which $y_{1}\left(t^{*}\right)=y_{2}\left(t^{*}\right)=0$, $y_{1}^{\prime}\left(t^{*}\right) \neq 0, y_{2}^{\prime}\left(t^{*}\right) \neq 0, y_{3}(t)=y(t)$, and $y_{i}(t) \neq 0$ for $i=1,2,3$ and $t>t^{*}$.

If $r_{12}=\infty$ and $\lim _{t \rightarrow \infty} y_{i}(t) / y_{j}(t)$ exists for all $i, j$ then, if the solution specified by $y(u)=y^{\prime}(u)=0$ tends to zero for $t \rightarrow \infty$ there exists a basis $\left(y_{1}, y_{2}, y_{3}=y\right)$ of the solutions for which $y_{1} / y_{3}$ is monotone and $y_{2} / y_{3}$ has at most one extremum in $(u, \infty)$. If $y$ does not tend to a limit 0 then there exists a basis for which $\lim _{t \rightarrow \infty} y_{1}(t)=\lim _{t \rightarrow \infty} y_{2}(t)=0$.

In the first case, let $\mathbf{y}^{a}(t)$ be the image of $\mathbf{y}(t)$ in the affine plane given by the tangent to $\mathbf{y}(u)$ as line at infinity $y_{3}=0$. Then $\mathbf{y}^{a}$ tends to a limit point or a limit curve in the affine plane. Since $y^{a}$ is locally convex and for $t>t^{*}$ is in one halfplane of the affine tangent to $\mathbf{y}^{a}\left(t^{*}\right)$, the distance of the set $\left\{\mathbf{y}^{a}(t)|t\rangle\right.$ $\left.t^{*}+\varepsilon\right\}$ from the backward halftangent at $\mathbf{y}^{a}\left(t^{*}\right)$ is positive, e.g. in the euclidean metric of the cartesian plane. Hence, there exists an angle of vertex $\mathbf{y}^{a}\left(t^{*}\right)$ so that no line in the not empty interior of the angle intersects $\mathbf{y}^{a}(t)$ for $t>t^{*}$. If $\mathbf{y}^{a}$ is given in a coordinate system for which the lines $y_{1}=y_{2}=0$ are distinct lines through $\mathbf{y}^{a}\left(t^{*}\right)$ in the interior of the angle, the coordinate functions have the desired properties.

If we apply the same construction to the case where $\lim y_{i} / y_{j}$ exists and $\lim y(t)=0, \mathbf{y}^{a}(t)$ is a curve in the affine plane with asymptotes for $t \downarrow u$ and $t \uparrow \infty$. In the natural euclidean metric, the angle between the negatively oriented first and positively oriented second asymptotes is $\leqslant \pi$. We take as line $y_{1}=0$ a normal to the first asymptote and as $y_{2}=0$ a parallel to the asymptote. If $y$ does not tend to a $\operatorname{limit}$ zero, $\lim y^{a}(t)$ is a point $P$ of the affine plane. Then we take $y_{1}=0$ and $y_{2}=0$ through $P$.

2. If it is assumed that $r_{12}(u)=0$, we transform $\mathbf{y}(t)$ by polarity. The polar curve $\mathbf{y}^{*}(t)$ represents the solutions of the adjoint equation [2], [5], [9]; this holds in $n$ dimensions. The condition $r_{n-11}=\infty$ means that no osculating hyperplane of $\mathbf{y}$ intersects the curve for later values of $t$ and $r_{1 n-1}=\infty$ means that no osculating hyperplane contains any points of the curve for earlier values of $t$. Since polarity for curves induces a correspondence between points and osculating hyperplanes [9], the statements are duals of one another (see [5] for $n=3$ ): 
Proposition 3. The adjoint of an nth order equation with $r_{1 n-1}=\infty$ is an equation with $r_{n-11}=\infty$ and vice versa.

As a consequence, we can prove

PROPOSITION 4. If $r_{12}=\infty$ for a third order linear differential equation then either $\lim _{t \rightarrow \infty} y_{i}(t) / y_{j}(t)$ exists for any two solutions of the equation or $\mathbf{y}(t)$ is asymptotic to a closed curve which is either a projectively convex curve or a straight line.

Since any two polarities are obtained from one another by a collineation, we need only consider polarity in an ellipse of an affine plane. The polar dual of an affine locally convex arc is an affine locally convex arc as long as no tangent passes through the center of the conic. Hence, the notion of a closed, projectively convex curve is self-dual. If a locally convex $C^{1+k}$ Jordan arc in an affine plane tends to a limit point, we take the limit point as the center of the ellipse. The dual curve in the projective plane is a smooth Jordan arc without cusps nor inflections whose tangents tend to the line at infinity (e.g., in the sense of Plücker coordinates). Since the projective plane is compact, the closure of the dual curve is the union of the dual curve and either a single point (if the given curve tends to its limit with a well defined tangent) or the line at infinity (if no limit direction exists). Proposition 4 enumerates all possibilities for the dual of a curve described in Proposition 1.

3. For $n$th order equations we treat first the case $r_{i_{1} \ldots i_{k} 1_{1}}=\infty, \sum i_{j}=n-$ 2. We choose $t_{1}<\cdots<t_{k}$ and take as (hyper)plane $y_{1}=0$ a plane which has contact of order $i_{j}$ with $\mathbf{y}(t)$ at $t=t_{j}$; the order of contact is counted as the order of the zero of the coordinate function $y_{1}(t)=0$. By hypothesis, the curve intersects the hyperplane at most one more time at $t^{*}$. For $t>t^{*}, y(t)$ is in the affine space $y_{1} \neq 0$ and $y_{1}(t) \neq 0$ for $t>t^{*}$. If $t^{*}$ does not exist, we complete the definition by taking $t^{*}=t_{k}$. We now fix another set of parameters $t^{*}<t_{1}^{*}<\cdots<t_{k}^{*}$. The hyperplanes having contact of order $i_{j}$ with $\mathbf{y}(t)$ at $t_{j}^{*}$ form a one-dimensional pencil that can be parametrized by the points of a projective line, i.e., an angle variable $\alpha$. No hyperplane in the pencil will intersect $\mathbf{y}(t)$ more than once for $t>t_{k}^{*}$, the exact order of contact of curve and hyperplane must be odd and we obtain a monotone one-to-one map of $\left(t_{k}^{*}, \infty\right)$ and a connected set $\{\alpha\} \subset S^{1}$. Hence, $\lim _{t \rightarrow \infty} \alpha(t)$ exists. The corresponding hyperplane is either $y_{1}=0$ or it may be taken as $y_{n}=0$. In the second case, $y_{n}(t) \neq 0$ for $t>t_{k}^{*}$ and $y_{n} \rightarrow 0$ for $t \rightarrow \infty$. The intersection of $\left\{y_{n}=0\right\}$ and $\operatorname{cl}\{\mathbf{y}(t)\}$ is not empty. A point in the intersection may be taken as vertex of a fundamental simplex for coordinates $\left\{y_{i}\right\}$. For $t \rightarrow \infty$, the functions $y_{i}(t), 1<i<n$, come arbitrarily close to 0 an infinity of times. In the first case, we take the vertex in $\left\{y_{1}=0\right\}$. Then $\lim _{t \rightarrow \infty} y_{1}(t)=0$ and the $y_{i}(t)$ come arbitrarily close to 0 an infinity of times for $1<i<n$. We have proved

Proposition 5. If $r_{i_{1} \ldots i_{k} 11}=\infty, \sum i_{j}=n-2$ then for any $t^{*}>t_{0}$ there 
exists a solution $y_{1}(t)$ of the equation which vanishes at $t^{*}$ and is different from zero for $t>t^{*}$.

If $\lim _{t \rightarrow \infty} y_{1}(t)=0$ then there exists a basis $\left\{y_{1}, \ldots, y_{n}\right\}$ of solutions for which $y_{2}(t), \ldots, y_{n-1}(t)$ come arbitrarily close to zero an infinity of times, i.e., for every $\varepsilon>0$ we have $\left|y_{i}(t)\right|<\varepsilon$ for infinitely many distinct values of $t>t^{*}$. If the limit of $y_{1}(t)$ either does not exist or is $\neq 0$, it is possible to find a basis with the same property and in addition $\lim _{t \rightarrow \infty} y_{n}(t)=0$.

For every solution $y(t)$ with preassigned orders $i_{1}, \ldots, i_{k}$ of $k$ zeros the exact order of the single following zero must be odd.

In the special case $r_{n-211}=0$, we can show that $\lim _{t \rightarrow \infty} \mathbf{y}(t)$ exists: In the proof of Proposition 5, put $I=\operatorname{cl}\{\mathbf{y}(t)\} \cap\left\{y_{i}=0\right\}$ where $i=1$ in the first case and $i=n$ in the second case. $I$ is a bounded set in an affine space of dimension $n-2$. Two parallel support spaces of dimension $n-3$ are cut out by hyperplanes in the $(n-1)$-space which pass through the osculating space of order $n-2$ at $y\left(t^{\prime}\right)$ for some $t^{\prime}>t_{k}$. If $y(t)$ has no limit, $I$ consists of at least two points, the hyperplanes are distinct and define a pencil of which an infinity of planes intersects the curve an infinity of times; contradiction.

Proposition 6. If $r_{n-211}=\infty$, there exists a basis of solutions which contains $n-1$ functions tending to 0 for $t \rightarrow \infty$.

To obtain Proposition 6 we choose $I$ as vertex of the fundamental simplex.

4. Next we study the case $r_{1 n-21}=\infty$ (see [7] for an investigation of $\left.r_{121}=\infty\right)$. We take the hyperplane through $\mathbf{y}\left(t_{0}\right)$. which has contact of order $n-2$ with the curve at $\mathbf{y}\left(t_{1}\right)$ as hyperplane at infinity; for $t>t_{1}$ the curve is in the affine space belonging to that hyperplane. We fix $t^{*}>t_{1}$. If $n=2 k$ is even, the curve will be in one closed halfspace of the hyperplane through $\mathbf{y}\left(t^{*}\right)$ which has contact of order $2 k-2$ with the curve at $\mathbf{y}(t)$, for all $t>t^{*}$. These hyperplanes therefore are support hyperplanes for $t^{\prime}>t$, of a locally convex cone of vertex $\mathbf{y}\left(t^{*}\right)$ and none of the hyperplanes can be osculating since for a curve with nonvanishing Wronskian the exact order of contact of the osculating plane is always $n-1$. For $n=4$ it follows that the generators of the cone intersect the plane at infinity in a locally convex Jordan curve, see $\S 1$.

Proposition 7. If $n$ is even, $r_{1 n-21}=\infty$ implies $r_{1 n-1}=\infty$. If $n=4$ and $r_{121}=\infty$ then the lines $\lambda_{1} \mathbf{y}\left(t^{*}\right)+\lambda_{2} \mathbf{y}(t)$ either tend to a limit or are asymptotic to the generators of a cone which intersects the plane at infinity in a closed, projectively convex curve.

\section{REFERENCES}

1. J. H. Barrett, Oscillation theory of ordinary linear differential equations, Advances in Math. 3 (1969), 415-509. MR 41 \#2113.

2. G. D. Birkhoff, On the solutions of ordinary linear homogeneous differential equations of the third order, Ann. of Math. 12 (1910-1911), 103-127. 
3. H. Guggenheimer, Homogeneous linear differential equations with only periodic solutions, Israel J. Math. 9 (1971), 49-52. MR 42 \#6352.

4. __ Geometric theory of differential equations. IV:Two-point boundary value problems of linear equations, Rend. Mat. (6) 5 (1972), 237-250.

5. M. Hanan, Oscillation criteria for third-order linear differential equations, Pacific J. Math. 11 (1961), 919-944. MR 26 \#2695.

6. F. Neuman, Geometrical approach to linear differential equations of the $n$-th order, Rend. Mat. (6) 5 (1972), 579-602. MR 48 \#2493.

7. J. R. Ridenhour and T. L. Sherman, Conjugate points for fourth order linear differential equations, SIAM J. Appl. Math. 22 (1972), 599-603. MR 46 \#2151.

8. B. Segre, Alcune proprietà differenziali in grande delle curve chiuse sghembe, Rend. Mat. (6) 1 (1968), 237-297. MR 39 \#4787.

9. E. J. Wilczynski, Projective differential geometry of curves and ruled surfaces, Teubner, Leipzig, 1905; Chelsea, New York.

Department of Mathematics, Polytechnic Institute of New York, Brooklyn, New YORK 11201 